\title{
Estudo experimental da influência da adição de microfibras de polipropileno e da cura na dureza superficial de pisos de concreto
}

\section{Experimental study of the influence of the addition of polypropylene microfibers and cure on the surface hardness of concrete floors}

Janaine Maria Golfetto ${ }^{1}$, Francine Padilha ${ }^{1}$, Beatriz Schimelfenig ${ }^{1}$, Cristina Vitorino da Silva ${ }^{1}$

\footnotetext{
${ }^{1}$ Universidade Regional Integrada do Alto Uruguai e das Missões - URI Erechim, RS-331, 99700-000, Erechim, Rio Grande do Sul, Brasil.

e-mail: jana.golfetto@ hotmail.com, beatrizschimelfenig@gmail.com, francine.padilha1609@gmail.com, cristina@uricer.edu.br
}

\section{RESUMO}

As falhas de dosagem e execução de pisos de concreto, constantemente levam ao surgimento de manifestações patológicas. Sendo assim, é indispensável o conhecimento dos fatores que influenciam o comportamento do concreto ou alteram suas características, bem como a utilização de mão de obra de qualidade. Em vista disso, este estudo avaliou a influência da adição de microfibras de polipropileno nos teores de $0 \mathrm{~g} / \mathrm{m}^{3}, 600$ $\mathrm{g} / \mathrm{m}^{3}$ e $900 \mathrm{~g} / \mathrm{m}^{3}$ e de diferentes condições de cura (sem cura, cura úmida e cura úmida após três dias de exposição ao ambiente) nos concretos empregados em pisos. Para tanto, foram realizados ensaios de exsudação, resistência à compressão uniaxial e dureza superficial por esclerometria. Os resultados indicam que a utilização das microfibras influenciou de forma significativa a exsudação e a resistência à compressão, ainda que a dureza superficial não tenha sido afetada diretamente por este material e nem pelo tipo de cura. De maneira geral, pode-se dizer que dentre os teores de microfibras de polipropileno avaliados, o teor de $600 \mathrm{~g} / \mathrm{m}^{3}$ é o mais indicado para adição no concreto, apresentando melhor desempenho nas propriedades analisadas. É possível afirmar ainda que a cura, apesar de não ter causado influência significativa na superfície do concreto, é um fator de extrema importância para suas propriedades de massa.

Palavras-chave: Pisos de Concreto; Manifestações Patológicas; Microfibras de Polipropileno; Cura; Dureza Superficial.

\begin{abstract}
Failures of dosing and execution of concrete floors, constantly lead to the emergence of pathological manifestations. Therefore, it is indispensable to know the factors that influence the behavior of the concrete or alter its characteristics, as well as the use of quality manpower. This study evaluated the influence of the addition of polypropylene microfibers in the contents of $0 \mathrm{~g} / \mathrm{m}^{3}, 600 \mathrm{~g} / \mathrm{m}^{3}$ and $900 \mathrm{~g} / \mathrm{m}^{3}$ and of different curing conditions (without cure, wet cure and wet cure after three days of exposure) in concrete used in floors. For that, tests of bleeding, compressive strength and surface hardness by rebound hammer were per-formed. The results indicate that the use of microfibers significantly influenced the bleeding and the compressive strength, although the surface hardness was not affected directly by this material nor by the type of cure. In general, it can be said that of the polypropylene microfiber contents evaluated, the $600 \mathrm{~g} / \mathrm{m}^{3}$ content is the most suitable for addition in the concrete, presenting better performance in the analyzed properties. It is possible to affirm still that the cure, although not having caused significant influence on the surface of the concrete, is a factor of extreme importance for its mass properties.
\end{abstract}

Keywords: Concrete Floors; Pathological Manifestations; Polypropylene Microfibers; Cure; Surface Hardness. 


\section{INTRODUÇÃO}

O concreto é um material utilizado em grande escala na construção civil. Desta forma, é de grande importância o seu estudo tecnológico, para que possa ser empregado com qualidade e segurança. Quando a aplicação do concreto se refere a pisos, as propriedades mais significativas são as resistências mecânicas e a dureza superficial. Porém, estas propriedades podem sofrer influência de diversos fatores, como as variações do meio em que o concreto está inserido, a escolha e dosagem dos materiais, o elevado consumo de água, as condições impróprias de cura, além de outras falhas no processo de execução [1]. Estes fatores contribuem para o surgimento de manifestações patológicas, dentre as quais estão as fissuras por retração, fenômeno comumente encontrado em pisos industriais de concreto. Tal fenômeno está diretamente ligado ao processo de cura, por isso é necessário que se tome cuidado para que essa etapa seja realizada de maneira correta. Outra manifestação patológica verificada com grande frequência em pisos é o desgaste superficial, causado pela baixa resistência mecânica, principalmente da camada superficial. Este fato é influenciado pela ocorrência da exsudação excessiva, sendo importante a dosagem correta dos materiais [2].

No intuito de minimizar a ocorrência destes problemas, vem surgindo diferentes alternativas e soluções inovadoras no mercado de pisos de concreto. No entanto, a evolução do conhecimento nesse mercado produtivo não acompanha o surgimento destas novas soluções, havendo, ainda, a necessidade de estudos científicos e tecnológicos nesta área. Portanto, o presente estudo pretende contribuir para o conhecimento do comportamento do concreto empregado em pisos, analisando a influência da cura e da adição de microfibras de polipropileno em suas propriedades.

\section{MATERIAIS E MÉTODOS}

Através deste estudo avaliou-se a influência da adição de microfibras de polipropileno nos teores de $0 \mathrm{~g} / \mathrm{m}^{3}$, $600 \mathrm{~g} / \mathrm{m}^{3}$ e $900 \mathrm{~g} / \mathrm{m}^{3}$ e de diferentes condições de cura (sem cura, cura úmida e cura úmida após três dias de exposição ao ambiente) em concretos com resistência característica à compressão (fck) de $30 \mathrm{MPa}$, empregados em pisos. Para tanto, foram realizados ensaios de exsudação, resistência à compressão uniaxial e dureza superficial por esclerometria. A Figura 1 apresenta o programa experimental proposto.

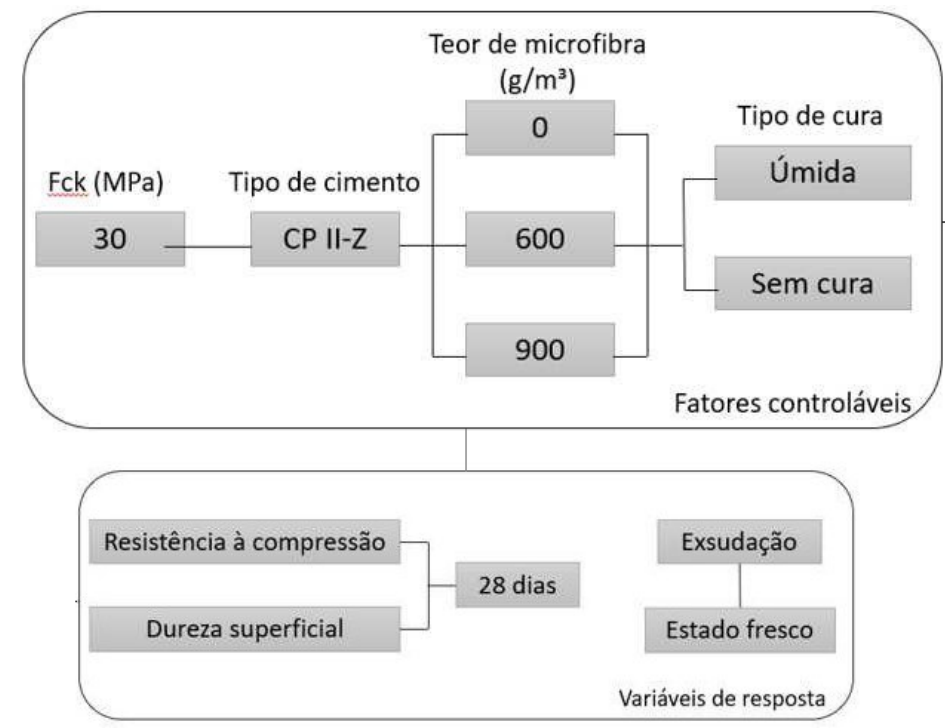

Figura 1: Programa experimental.

\subsection{Materiais}

Os concretos foram produzidos com cimento CP II-Z, por ser o tipo mais empregado nas obras de pisos na região de Erechim-RS, agregado miúdo natural de origem quartzosa e agregado graúdo de origem basáltica, contido na zona granulométrica 9,5/25 (brita 1). Para melhorar a dispersão das microfibras, bem como a trabalhabilidade da mistura, adicionou-se aditivo superplastificante. As características dos materiais utilizados são mostradas na Tabela 1. 
Tabela 1: Principais características dos materiais utilizados.

\begin{tabular}{c|l|l|l|l}
\hline & \multicolumn{1}{|c|}{ CIMENTO } & \multicolumn{1}{|c|}{$\begin{array}{c}\text { AGRAGADO } \\
\text { MIÚDO }\end{array}$} & \multicolumn{1}{c}{$\begin{array}{c}\text { AGREGADO } \\
\text { GRAÚDO }\end{array}$} & \multicolumn{1}{c}{ MICROFIBRAS } \\
\hline MASSA ESPECÍFICA $\left(\mathbf{g} / \mathbf{c m}^{3}\right)$ & 2,96 & 2,60 & 2,57 & 0,91 \\
\hline MÓDULO DE FINURA & - & 1,47 & 6,91 & - \\
\hline DIMENSÃO MÁXIMA (mm) & - & 1,18 & 19 & - \\
\hline ABSORÇÃO DE ÁGUA (\%) & - & 0,8 & 2,98 & - \\
\hline COMPRIMENTO $(\mathbf{m m})$ & - & - & - & 12 \\
\hline DIÂMETRO $(\boldsymbol{\mu m})$ & - & - & - & 18 \\
\hline ALONGAMENTO $(\%)$ & - & - & - & 80 \\
\hline
\end{tabular}

\subsection{Dosagem}

A dosagem foi realizada pelo método do IPT/EPUSP, proposto por HELENE e TERZIAN [3], fixando-se o abatimento do concreto em $100 \pm 20 \mathrm{~mm}$, e o teor ideal de argamassa $(\alpha)$, em $54 \%$, determinado experimentalmente. A dosagem experimental está apresentada na Tabela 2.

Tabela 2: Dosagem experimental.

\begin{tabular}{l|l|l|l|l|l}
\hline $\begin{array}{c}\text { TRAÇO UNITÁRIO } \\
(\mathbf{1}: \mathbf{m})\end{array}$ & $\begin{array}{c}\text { RELAÇÃO } \\
\text { ÁGUA/CIMENTO } \\
(\mathbf{a} / \mathbf{c})\end{array}$ & $\begin{array}{c}\text { RESISTÊNCIA } \\
\mathbf{2 8} \text { DIAS } \\
\mathbf{( M P a )}\end{array}$ & $\begin{array}{c}\text { CONSUMO DE } \\
\text { CIMENTO } \\
\left(\mathbf{k g} / \mathbf{m}^{3}\right)\end{array}$ & $\begin{array}{c}\text { TEOR DE } \\
\text { UMIDADE } \\
(\%)\end{array}$ & $\begin{array}{c}\text { ABATIMENTO } \\
(\mathbf{m m})\end{array}$ \\
\hline $1: 3,5$ & 0,44 & 35,61 & 466,53 & 9,8 & 80 \\
\hline $1: 5,0$ & 0,64 & 23,29 & 342,74 & 10,7 & 130 \\
\hline $1: 6,5$ & 0,76 & 16,37 & 276,60 & 10,1 & 100 \\
\hline
\end{tabular}

\subsection{Ensaio de exsudação}

O ensaio de exsudação, realizado com o concreto no estado fresco, seguiu as prescrições da NBR 15558 [4]. No entanto, o molde para confecção dos corpos de prova foi em PVC, com dimensões reduzidas (200x150 $\mathrm{mm}$ ), mas respeitando-se a relação entre diâmetro e altura proposta pela Norma. Durante o ensaio os corpos de prova permaneceram em câmara climatizada (UR $60 \pm 10 \%$ e temperatura de $23 \pm 2^{\circ} \mathrm{C}$ ).

\subsection{Ensaio de compressão uniaxial}

O ensaio de resistência à compressão uniaxial seguiu a NBR 5739 [5]. Foram moldados, e ensaiados aos 28 dias, corpos de prova cilíndricos com dimensão 100x200 mm. Estes foram adensados em mesa vibratória e permaneceram cobertos por uma lona plástica durante as primeiras 24 horas, a fim de evitar a perda de água do concreto para o ambiente externo. Posteriormente, os corpos de prova foram desmoldados e mantidos em câmara úmida (UR $\geq 95 \%$ e temperatura de $23 \pm 2^{\circ} \mathrm{C}$ ) até a realização do ensaio.

\subsection{Ensaio de dureza superficial}

O ensaio de dureza superficial foi realizado conforme a NBR 7584 [6]. Foram moldadas placas de concreto com dimensões de 250x350x100 mm, marcando-se 16 pontos de leitura na superfície de cada uma, espaçados entre si $30 \mathrm{~mm}$, e com mais de $50 \mathrm{~mm}$ de distância das bordas. Para a realização do ensaio, as placas foram fixadas por duas de suas extremidades na prensa hidráulica, com aplicação de $30 \%$ da tensão de ruptura à compressão do concreto. A leitura dos pontos foi feita pelo esclerômetro de reflexão.

\section{RESULTADOS E DISCUSSÕES}

Os resultados obtidos nos ensaios são apresentados e discutidos a seguir.

\subsection{Exsudação}

O percentual de água exsudada durante o ensaio é mostrado na Tabela 3. 
Tabela 3: Resultados do ensaio de exsudação.

\begin{tabular}{c|c|c}
\hline TRAÇO & $\begin{array}{c}\text { TEOR DE MICROFIBRA } \\
\left(\mathbf{g} / \mathbf{m}^{\mathbf{3}}\right)\end{array}$ & $\begin{array}{c}\text { EXSUDAÇÃO } \\
\mathbf{( \% )}\end{array}$ \\
\hline 1 & 0 & 2,067 \\
\hline 2 & 600 & 3,736 \\
\hline 3 & 900 & 3,895 \\
\hline
\end{tabular}

A exsudação aumentou em $80,7 \%$ para a adição de $600 \mathrm{~g} / \mathrm{m}^{3}$ de microfibra e $88,4 \%$ para a adição de 900 $\mathrm{g} / \mathrm{m}^{3}$, como pode ser visualizado também na Figura 2.

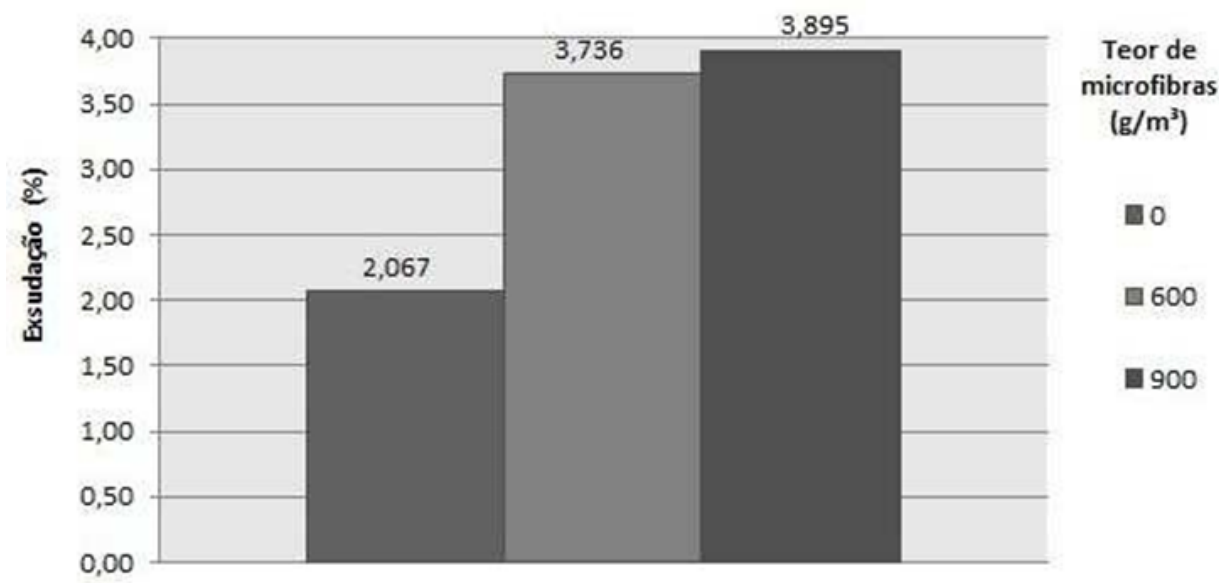

Figura 2: Quantidade de água exsudada, em percentual.

A orientação dos filamentos de microfibra pode ter sido a responsável pela maior quantidade de água exsudada no concreto, através da criação de caminhos preferenciais para sua saída. No entanto, essa orientação somente poderia ser confirmada por meio de análise microscópica do compósito cimentício. Com isso, não se pôde determinar se tal material exerce influência nesta propriedade, conforme revelado também nos estudos de SILVA [7]. Para isso, seria necessário aumentar o número de amostras ensaiadas, obtendo assim resultados mais precisos.

\subsection{Resistência à compressão uniaxial}

Os resultados do ensaio de compressão uniaxial são apresentados na Tabela 4. Estes resultados foram analisados estatisticamente pelo método da análise de variância (ANOVA), para assim determinar a influência significativa ou não das microfibras, conforme a Tabela 5.

Tabela 4: Resultados do ensaio de resistência à compressão.

RESISTÊNCIA À COMPRESSÃO UNIAXIAL

\begin{tabular}{c|c|c|c|c}
\hline TRAÇO & $\begin{array}{c}\text { TEOR DE MICROFIBRA } \\
\left(\mathbf{g} / \mathbf{m}^{\mathbf{3}}\right)\end{array}$ & $\begin{array}{c}\text { MÉDIAS } \\
\mathbf{( M P a )}\end{array}$ & $\begin{array}{c}\text { DESVIO PADRÃO } \\
\mathbf{( M P a )}\end{array}$ & CV (\%) \\
\hline 1 & 0 & 29,21 & 0,38 & 1,31 \\
\hline 2 & 600 & 38,97 & 1,57 & 4,02 \\
\hline 3 & 900 & 35,61 & 1,32 & 3,70 \\
\hline \multicolumn{4}{r}{ CV coeficiente de variação. } \\
\hline
\end{tabular}


Tabela 5: Análise de variância para resistência de compressão uniaxial.

\begin{tabular}{c|c|c|c|c|c}
\hline & GDL & MS & F & P & SIGNIFICÂNCIA \\
\hline TEOR DE MICROFIBRA & 2 & 63,55 & 34,923 & 0,000228 & S \\
\hline ERRO & 7 & 1,82 & & & \\
\hline GLD = graus de liberdade; MS = média simples; F = valor calculado de F; \\
P = nível de significância; S = valor significativo; se P<5\%, valor significativo.
\end{tabular}

Através da análise percebe-se que a adição de microfibra influenciou significativamente a resistência à compressão, o que pode ser melhor visualizado na Figura 3.

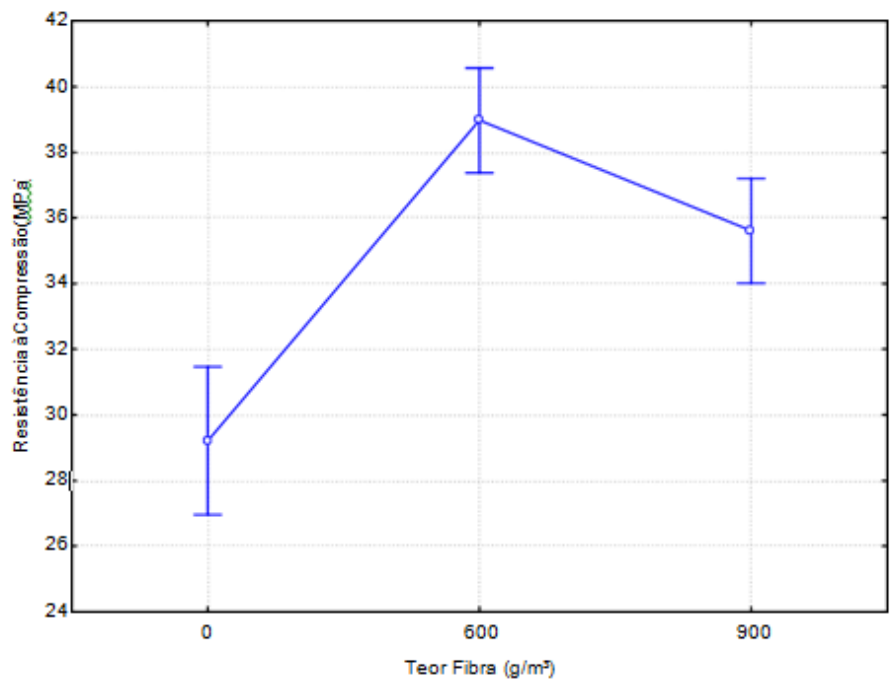

Figura 3: Efeito do teor de microfibra na resistência à compressão uniaxial do concreto.

Observa-se que a adição de microfibra elevou a resistência do concreto em 33,4\% e 25,3\% para os teores de $600 \mathrm{~g} / \mathrm{m}^{3}$ e $900 \mathrm{~g} / \mathrm{m}^{3}$, respectivamente. Tal fato não era esperado, pois o material possui baixo módulo de elasticidade não exercendo, desta forma, influência significativa nas propriedades mecânicas, o que já é confirmado em diversos estudos, como o de SILVA [7] e o de RICHARDSON [8], que avaliou a resistência à compressão de concretos com adição de $450 \mathrm{~g} / \mathrm{m}^{3}, 900 \mathrm{~g} / \mathrm{m}^{3}$ e $1800 \mathrm{~g} / \mathrm{m}^{3}$. Contudo, para os materiais e teor de aditivo utilizado neste estudo, as microfibras podem ter atuado como pontos de nucleação, retendo maior quantidade de água na zona de transição, e possibilitando, assim, maior hidratação das partículas de cimento presentes nessa zona, o que melhorou a coesão e resultou em ganhos de resistência. Além do mais, as microfibras tem a capacidade de amarrar as microfissuras iniciais do concreto, o que também pode ter contribuído para o aumento da resistência, através do retardamento da ruptura dos corpos de prova, como o ocorrido no estudo de SENISSE [9] e JUNIOR e ROCHA [10].

\subsection{Dureza Superficial}

A dureza superficial média verificada para cada condição ensaiada é apresentada na Tabela 6. 
Tabela 6: Resultados do ensaio de dureza superficial.

\begin{tabular}{|c|c|c|c|c|c|}
\hline \multicolumn{6}{|c|}{ DUREZA SUPERFICIAL } \\
\hline TRAÇO & $\begin{array}{c}\text { TEOR DE } \\
\text { MICROFIBRAS } \\
\left(\mathbf{g} / \mathbf{m}^{3}\right) \\
\end{array}$ & CONDIÇÃO DE CURA & IE MÉDIO & $\begin{array}{l}\text { DESVIO } \\
\text { PADRÃOO }\end{array}$ & $\begin{array}{l}\text { CV } \\
\text { (\%) }\end{array}$ \\
\hline $1-\mathrm{A}$ & 0 & úmida & 51,8 & 2,1 & 4,0 \\
\hline $1-\mathrm{B}$ & 0 & úmida após 3 dias & 51,4 & 2,4 & 4,7 \\
\hline $1-\mathrm{C}$ & 0 & sem & 50,5 & 0,5 & 1,0 \\
\hline $2-\mathrm{A}$ & 600 & úmida & 47,9 & 2,1 & 4,4 \\
\hline $2-\mathrm{B}$ & 600 & úmida após 3 dias & 53,7 & 1,5 & 2,9 \\
\hline $2-\mathrm{C}$ & 600 & sem & 51,2 & 0,5 & 1,0 \\
\hline $3-\mathrm{A}$ & 900 & úmida & 49,2 & 1,3 & 2,6 \\
\hline $3-\mathrm{B}$ & 900 & úmida após 3 dias & 51,1 & 3,4 & 6,6 \\
\hline $3-\mathrm{C}$ & 900 & sem & 50,7 & 2,2 & 4,3 \\
\hline
\end{tabular}

IE = índice esclerométrico; CV = coeficiente de variação.

Os dados foram analisados estatisticamente pelo método de análise da variância (ANOVA), o qual buscou determinar se o teor de microfibras, a condição de cura e a interação entre esses dois fatores teve influência significativa na propriedade avaliada, de acordo com a Tabela 7.

Tabela 7: Análise de variância para dureza superficial.

\begin{tabular}{c|c|c|c|c|c}
\hline & GDL & MS & F & P & SIGNIFICÂNCIA \\
\hline TEOR DE MICROFIBRA (1) & 2 & 1,17 & 0,30 & 0,747612 & NS \\
\hline TIPO DE CURA (2) & 2 & 9,02 & 2,31 & 0,155386 & NS \\
\hline (1) X (2) & 4 & 5,43 & 1,39 & 0,312552 & NS \\
\hline ERRO & 9 & 3,91 & & & \\
\hline
\end{tabular}

$\mathrm{GLD}=$ graus de liberdade; $\mathrm{MS}=$ média simples; $\mathrm{F}=$ valor calculado de $\mathrm{F}$;

$\mathrm{P}=$ nível de significância; $\mathrm{S}=$ valor significativo; se $\mathrm{P}<5 \%$, valor significativo.

Observa-se que nenhuma das variáveis de controle, nem a interação entre elas, tiveram efeito significativo sobre a dureza superficial. Sendo a dureza uma propriedade de superfície, o ensaio de esclerometria avalia o concreto apenas a uma profundidade de aproximadamente $3 \mathrm{~mm}$. Assim, de acordo com MEHTA e MONTEIRO [11], durante o ensaio o êmbolo pode impactar sobre agregados que ficam próximos à superfície, o que gera interferência nos resultados, uma vez que a dureza do agregado é superior à dureza da argamassa. A manifestação da real influência das variáveis de controle pode ter sido inibida por tal fator.

Porém, sabe-se que a cura é de extrema importância no desempenho do concreto, o que já foi comprovado em diversas pesquisas, como a de SILVA [1]. A autora avaliou a influência da cura submersa e em caixa aquecida na dureza superficial de concretos, confirmando o aumento de $3 \%$ para corpos de prova que receberam cura submersa. A inibição da influência da cura, nesse caso, ocorreu devido à ação das microfibras, que diminuindo a retração na fase plástica minimizou a abertura de fissuras nos concretos sem cura, igualando sua dureza superficial à dos concretos submetidos à cura úmida. Deste modo, o efeito da cura provavelmente seja mais notório nas propriedades de massa, como resistência à compressão, do que nas propriedades de superfície, uma vez que os estudos de SILVA et al. [12] e BAUER et al. [13] comprovaram um aumento significativo na resistência à compressão para corpos de prova que foram curados em câmara úmida ou submersos, em relação aos sem cura.

\section{CONCLUSÕES}

O percentual de exsudação foi significativamente maior com a presença das microfibras, que apesar de serem hidrofóbicas, formaram caminhos preferenciais para a saída da água do concreto. Com relação à resistência à compressão, as microfibras elevaram de maneira significativa o parâmetro, amarrando as microfissuras e atuando como pontos de nucleação. Na dureza superficial, no entanto, não se pôde observar influência direta das microfibras e nem da cura, por esta ser uma propriedade de superfície. Analisando-se de forma geral os parâmetros avaliados, pode-se indicar a adição de microfibras no teor de $600 \mathrm{~g} / \mathrm{m}^{3} \mathrm{como}$ a mais eficiente para utilização nos concretos empregados em pisos. 


\section{BIBLIOGRAFIA}

[1] SILVA, C. V., Estudo da influência das propriedades relacionadas à superfície e à matriz na resistência à abrasão de concretos para pisos, Tese de D. Sc., NORIE/UFRGS, Porto Alegre, RS, Brasil, 2015.

[2] CHODOUNSKY, M. A, Patologias em Pisos Industriais, Rio de Janeiro, ANAPRE, 2010.

[3] HELENE, P., TERZIAN, P, Manual de dosagem e controle do concreto, 1 ed., São Paulo, PINI, 1992.

[4] ASSOCIAÇÃO BRASILEIRA DE NORMAS TÉCNICAS, NBR 15558: Concreto - Determinação da exsudação, Rio de Janeiro, 2008.

[5] ASSOCIAÇÃO BRASILEIRA DE NORMAS TÉCNICAS, NBR 5739: Concreto - Ensaio de compressão de corpos de prova cilíndricos, Rio de Janeiro, 2007.

[6] ASSOCIAÇÃO BRASILEIRA DE NORMAS TÉCNICAS, NBR 7584: Concreto endurecido - Avaliação da dureza superficial pelo esclerômetro de reflexão, Rio de Janeiro, 2012.

[7] SILVA, C. V., Contribuição ao estudo do desgaste superficial por abrasão em concretos empregados em pisos, Tese de M. Sc., NORIE/UFRGS, Porto Alegre, RS, Brasil, 2011.

[8] RICHARDSON, A. E., "Compressive strength of concrete with polypropylene fiber additions", In: Structural Survey, v. 24, n. 2, p. 138-153, 2006.

[9] SENISSE, J. A. L., Influência do consumo de água, do teor de adição de microfibras de polipropileno e do tipo de cimento no fenômeno da retração por secagem em concretos empregados em pisos, Tese M. Sc., NORIE/UFRGS, Porto Alegre, RS, Brasil, 2010.

[10] JUNIOR, E. S.D., ROCHA, L. F. S., Estudo de concreto com adição de fibra de polipropileno para controle da fissuração, Trabalho de Conclusão de Curso, UNAMA, Belém, PA, Brasil, 2011.

[11] MEHTA, P. K., MONTEIRO, P. J., Concreto: microestrutura, propriedades e materiais, 3 ed., São Paulo, IBRACON, 2011.

[12] SILVA, A. V. R., DINIZ, H. A. A., RIBEIRO, J. A. E., et al., "Influência do processo de cura em concreto convencional em seis idades”, In: Congresso Norte-Nordeste de Pesquisa e Inovação, Palmas, Brasil, 2012.

[13] BAUER, R. J. F., CURTI, R., MARTINS, A., et al., "Estudo de característica física e mecânica do concreto pelo efeito de vários tipos de cura", In: L. A. Falcão Bauer Centro Tecnológico de Controle da Qualidade Ltda, São Paulo, Brasil, 2000.

\section{ORCID}

$\begin{array}{ll}\text { Janaine Maria Golfetto } & \text { https://orcid.org/0000-0002-2802-0391 } \\ \text { Francine Padilha } & \text { https://orcid.org/0000-0001-8662-628X } \\ \text { Beatriz Schimelfenig } & \text { https://orcid.org/0000-0002-1930-2055 } \\ \text { Cristina Vitorino da Silva } & \text { https://orcid.org/0000-0002-3073-676X }\end{array}$ 